\title{
Distal upper extremity musculoskeletal risk factors associated with colonoscopy
}

\author{
David Rempel ${ }^{\mathrm{a},{ }^{*}}$, David Lee ${ }^{\mathrm{a}}$ and Amandeep Shergill ${ }^{\mathrm{b}}$ \\ ${ }^{a}$ Department of Medicine, University of California, Ergonomics Program, Richmond Field Station, 1301 S. $46^{\text {th }}$ \\ Street, Building 163, Richmond, CA 94707, USA. \\ ${ }^{\mathrm{b}}$ Department of Gastroenterology, VA Hospital, San Francisco, CA, USA.
}

\begin{abstract}
Gastroenterologists are at increased risk for developing recurrent thumb, hand, and elbow pain due to colonoscopy procedures. We evaluated forearm muscle loads and wrist postures during routine colonoscopy ( $\mathrm{N}=12$ gastroenterologists) to understand distal upper extremity musculoskeletal risk factors associated with the 4 different subtasks of colonoscopy. Bilateral forearm extensor carpi radialis (ECR) and flexor digitorum superficialis (FDS) surface electromyography and bilateral wrist postures were recorded continuously. The mean duration of colonoscopy was $24.2( \pm 12.1)$ minutes and was dominated by the withdrawal subtask $[13.7( \pm 8.8) \mathrm{min}]$ followed by right colon insertion $[5.8( \pm 4.8) \mathrm{min}]$, left colon insertion $[3.5( \pm 3.1) \mathrm{min}]$, and retroflexion $[1.2( \pm 2.1) \mathrm{min}]$. Median (APDF50) and peak (APDF90) left forearm muscle activity was significantly greater than right forearm muscle activity across all subtasks. Median and peak ECR muscle activity was significantly greater during the left and right colon insertion subtasks compared to retroflexion. Both wrists were predominantly in wrist extension during all phases of colonoscopy. The left forearm muscle activity was higher than right forearm activity due to differences in wrist posture and grip force. The risk factors for the left hand may be reduced with alternative designs and support mechanisms for the colonoscope head.
\end{abstract}

Keywords: Gastroenterology, Ergonomics, Wrist, Electromyography, Tool Design

\section{Introduction}

Physicians who perform endoscopies are at increased risk for developing recurrent thumb, hand, and elbow pain. These injuries include wrist tendinopathies, epicondylitis, carpal tunnel syndrome, and shoulder or cervical muscle strains. These may present as hand numbness or pain in the hand, wrist, elbow, neck, or back [1,2]. Almost $60 \%$ of endoscopists surveyed have suffered some musculoskeletal complaint related to endoscopy, and higher endoscopy volume is associated with a higher likelihood of overuse injury [3].

Previously, we conducted a pilot study $(\mathrm{N}=9: 3$ gastroenterologists; 3 cases each) to evaluate the pinch forces and forearm muscle activity during colonoscopy [4]. The mean peak pinch forces on the left were $10.4 \mathrm{~N}(4.1)$ and on the right were $10.1 \mathrm{~N}$
(4.5). Peak forearm muscle activity was greater on the left than the right. In all cases, the colonoscopy head was held with the left hand. The left hand grips and stabilizes the control section and the left thumb rotates the control dials, resulting in tip deflection of the insertion tube (see Figure 1). The index and long finger on the left hand can aid with dial rotation, but are primarily used to activate water, air and suction buttons on the control section. The right hand pinches or grips the insertion tube, and the right arm pushes, pulls, and applies torque to the endoscope during insertion and withdrawal (see Figure 2).

A typical, simple colonoscopy procedure has 4 subtasks: insertion of the colonoscope into the left colon, insertion into the right colon, withdrawal and retroflexion. The procedure becomes more complicated if biopsies are taken, a polypectomy is performed or the colon preparation is suboptimal.

\footnotetext{
*Corresponding author. E-mail: david.rempel@ucsf.edu.
} 
In the current study, we expanded the prior study by evaluating more gastroenterologists $(\mathrm{N}=12)$ and by also measuring wrist posture during colonoscopy to improve our understanding the distal upper extremity musculoskeletal risk factors associated with the different subtasks of colonoscopy.

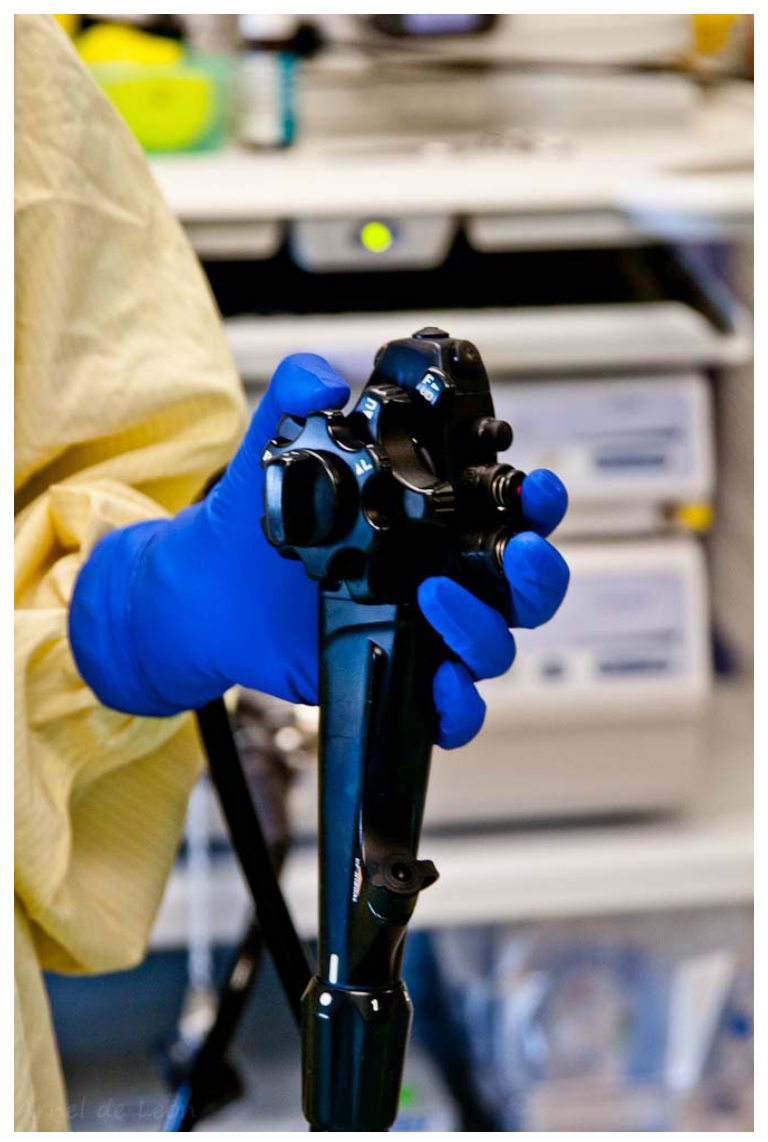

Figure 1. Left hand holding control section

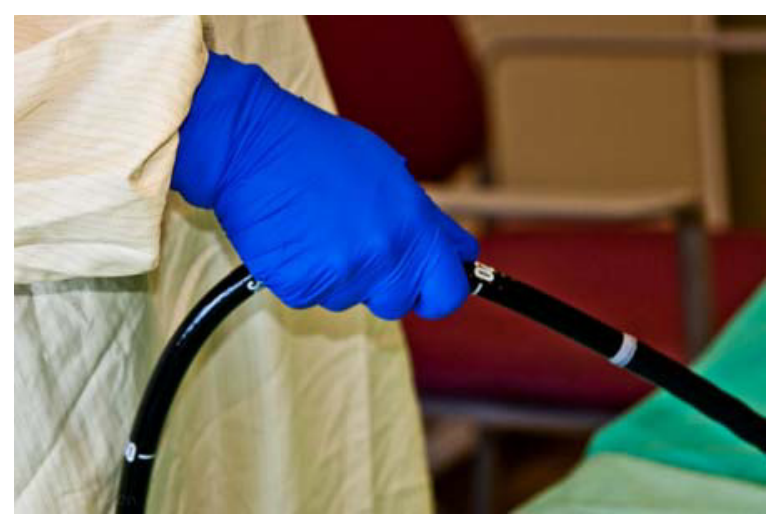

Figure 2. Right hand holding insertion tube

\section{Methods}

Gastroenterologists from the San Francisco VA Medical Center and the University of California at San Francisco with more than 4 years experience performing endoscopies were recruited to the study. The participants were free of recurrent upper extremity pain or disorders. The endoscopists as well as the patients undergoing colonoscopy provided informed consent. The study was approved by the Investigational Review Boards of the University of California, San Francisco and the San Francisco Veterans Affairs Medical Center.

Bilateral forearm extensor carpi radialis (ECR) and flexor digitorum superficialis (FDS) surface electromyography and bilateral wrist postures were recorded continuously (TeleMyo 2400T, Noraxon USA Inc, Scottsdale, AZ) (see Table 1). EMG signals were preamplified, passed through a $1^{\text {st }}$ order high pass filter set to a $10 \mathrm{~Hz}$ cutoff and a Butterworth low pass antialias filter set to a $1000 \mathrm{~Hz}$ cutoff. Data were sampled at $1500 \mathrm{~Hz}$ and recorded to a personal computer using MyoResearch XP software (Noraxon USA Inc., Scottsdale, Az). To standardize the EMG data, maximum voluntary electrical activity (MVE) was obtained by having the endoscopist perform a 5 second isometric maximum voluntary contraction (MVC) for each muscle. Electrogoniometers were attached to the dorsum of both wrists and calibrated prior to data collection.

Twelve gastroenterologists were studied while each performed 2 to 5 routine colonoscopies. A second gastroenterologist independently observed the colonoscopy and recorded insertion time, withdrawal time, time needed for polypectomies, and time spent in retroflexion in the rectum. Left-colon and rightcolon insertion times were defined as the advancement time from rectum to splenic flexure and from splenic flexure to cecum, respectively. Retroflexion in the rectum was considered separately because of the increased upper extremity forces required for this activity. Withdrawal time excluded time spent in retroflexion.

Summary measures of EMG and posture data were calculated using the amplitude probability distribution function (APDF). Repeated measures ANOVA was used to evaluate differences between hands and subtasks. Significant findings were followed up with the Tukey test to adjust for multiple comparisons. 


\section{Results}

The mean duration (SD) of colonoscopy was 24.2 $( \pm 12.1)$ minutes and was dominated by the withdrawal subtask [13.7 $( \pm 8.8) \mathrm{min}]$ followed by right colon insertion [5.8 $( \pm 4.8) \mathrm{min}]$, left colon insertion [3.5 $( \pm$ 3.1) $\mathrm{min}]$, and retroflexion $[1.2( \pm 2.1) \mathrm{min}]$. In all cases the left hand manipulated the colonoscope head while the right hand manipulated the insertion tube. Median (APDF50) and peak (APDF90) left forearm muscle activity was significantly greater than right forearm muscle activity across all subtasks (see Fig 3, 4). Median and peak ECR muscle activity was significantly greater during the left and right colon insertion subtasks compared to retroflexion. Both the right and left wrists were predominantly in wrist extension during all phases of colonoscopy (see Figure 5). The left wrist was predominantly in radially deviation while the right wrist was predominantly in ulnar deviation (see Figure 6).

\section{Conclusions}

During colonoscopy the left forearm muscle activity was higher than right forearm activity due to differences in hand activity, grip force, and increased wrist radial deviation. The left forearm is active in grasping and stabilizing the control section of the colonoscope. Repeated, high left ECR muscle loads may lead to second and third extensor compartment tenosynovitis at the wrist and epicondylitis. The risk factors for the left hand may be reduced with alternative designs and support mechanisms for the colonoscope head.

\section{References}

[1] Liberman, A.S., I. Shrier, and P.H. Gordon, Injuries sustained by colorectal surgeons performing colonoscopy. Surg Endosc, 2005. 19(12):1606-9.

[2] Cappell, M.S., Colonoscopist's thumb: DeQuervains's syndrome (tenosynovitis of the left thumb) associated with overuse during endoscopy. Gastrointest Endosc, 2006. 64(5):841-3

[3] Buschbacher, R., Overuse syndromes among endoscopists. Endoscopy, 1994. 26(6):539-44.

[4] Shergill AA, KR; Barr, A; Shah, JN; Ryan, JC; McQuaid, KR; Rempel, D. Pinch Force and Forearm Muscle Load During Colonoscopy: A Pilot Study. Gastrointestinal Endoscopy 2009, 69(1):142-6. 